\title{
Atrial Natriuretic Factor Gene Expression in Ventricles of Rats with Spontaneous Biventricular Hypertrophy
}

\author{
Richard T. Lee, Kenneth D. Bloch, Janice M. Pfeffer, Marc A. Pfeffer, Eva J. Neer, and Christine E. Seidman \\ Cardiovascular Division, Department of Medicine, Brigham and Women's Hospital and the Department of Genetics, \\ Harvard Medical School, Boston, Massachusetts 02115
}

\begin{abstract}
A subset of Wistar-Kyoto (WKY) rats that spontaneously develops biventricular hypertrophy (BVH) in response to increased cardiac output was evaluated for ventricular expression of the atrial natriuretic factor (ANF) gene. Normal WKY rats had low levels of left ventricular ANF mRNA and minimally detectable ANF transcripts in the right ventricle. In contrast, BVH rats showed a sixfold greater ANF mRNA concentration in the left ventricle than age-matched WKY controls. BVH right ventricular ANF mRNA levels equaled those found in BVH left ventricles and were dramatically greater than WKY right ventricular controls. Unlike experimental models of hypertrophy, both left and right ventricles significantly increase ANF gene transcripts in the natural development of BVH. The left and right ventricles can concordantly respond to hypertrophy and increase ANF gene transcription.
\end{abstract}

\section{Introduction}

Atrial natriuretic factor (ANF) ${ }^{1}$ a potent natriuretic and vasorelaxant cardiac hormone that inhibits renin and aldosterone secretion, is synthesized and secreted predominantly by the atria in healthy adult mammals (1-3). Small quantities of ANF mRNA have been detected in nonatrial tissues, particularly the ventricles, where levels approximate $1 / 50$ to $1 / 250$ of atrial concentrations in euvolemic animals $(4,5)$. Increased ANF gene expression in the ventricle has been demonstrated in animal models producing cardiac hypertrophy. These models include surgically produced aortic constriction (6), administration of high dose pharmacologic agents (6), or sudden circulatory overload due to ureteral constriction, nephrectomy, or arteriovenous shunting (7). These procedures produce dramatic changes in cardiovascular hemodynamics and analysis of ANF gene transcription following brief experimental periods has documented marked increases predominantly in the left ventricle (LV). Only one model has demonstrated increases in right ventricular gene expression (7), at levels far

Address reprint requests to Dr. Lee, Cardiovascular Division, Department of Medicine, Brigham and Women's Hospital, 75 Francis Street, Boston, MA 02115.

Received for publication 8 July 1987 and in revised form 14 September 1987.

1. Abbreviations used in this paper: ANF, atrial natriuretic factor; BVH, biventricular hypertrophy; iANF, immunoreactive ANF; LV, left ventricle; WKY, Wistar-Kyoto rat.

J. Clin. Invest.

(c) The American Society for Clinical Investigation, Inc.

0021-9738/88/02/0431/04 \$2.00

Volume 81, February 1988, 431-434 below that seen in the hypertrophied left ventricle. Localization of ANF gene induction primarily to one ventricular chamber may occur as an adaptive response to acute hemodynamic stress placed on the $L V$, represent nonspecific gene activation associated with chamber hypertrophy, or be a consequence of the model systems examined to date.

We have studied ANF gene expression in a unique model of naturally occurring biventricular hypertrophy (BVH) in a subset of Wistar-Kyoto rats (WKY). Unlike other models of cardiac hypertrophy, this condition is spontaneous, gradual, and progressive, permitting the animal to develop multiple compensatory mechanisms to mitigate the pathophysiologic sequelae of BVH. Animals develop a high output cardiac state with increased cardiac index, but retain normal blood pressure and have lowered peripheral resistance. Antemortum diagnosis of this condition is difficult, in part due to adaptive responses which prevent distinction of affected animals from age-matched WKY controls. Cardiac catheterization has been used to define BVH animals, and shows a hyperdynamic state (8). Postmortem evaluation of these rats reveals striking biventricular hypertrophy with right and left ventricles weighing up to twice normal.

Ventricular ANF gene expression increases in these animals despite normal blood pressure and lowered peripheral resistance. However in contrast to other interventions causing cardiac hypertrophy, spontaneous BVH induces high levels of ANF mRNA in both the right and left ventricle. These data demonstrate that both ventricular chambers can produce this peptide, and suggest that gene expression correlates with the hypertrophied state of each chamber.

\section{Methods}

Animals. Male WKY rats, ranging in age from 9 to $14 \mathrm{mo}$, were obtained from our colony. A small subset of WKY rats $(\sim 10 \%)$ will develop biventricular hypertrophy (8). Simple premortem tests for selecting these animals are unavailable. By random sacrifice of animals, we identified three BVH animals, based on ventricular to body weight ratios. One of the control animals (WKY 1) was from the same litter as a BVH animal (BVH 2). Animals were anesthetized with ether and hearts were excised. The atria and free wall of the right ventricle were separated and weighed individually; the interventricular septum was considered part of the left ventricle. The fibrous atrioventricular septum was removed from the left ventricle to assure that no contaminating atrial tissue was present. Atrial and ventricular tissues were homogenized in $4 \mathrm{M}$ guanidium isothiocyanate.

RNA characterization. RNA was prepared by the guanidium isothiocyanate-cesium chloride method (9) and quantitated by measuring absorbance at $260 \mathrm{~nm}$. RNA was electrophoresed in formaldehydeagarose gels and transferred to Genescreen Plus (NEN Products, Boston, MA). Northern analysis was performed using a ${ }^{32} \mathrm{P}$-labeled Pst 1 restriction fragment derived from a rat ANF cDNA as previously described (10). ANF probe was removed from the filters by boiling. To quantitate the amount of RNA loaded on agarose gels, a ${ }^{32} \mathrm{P}$-labeled 
oligonucleotide probe [5'-ACGGTATCTGATCGTCTTCGAACC-3'] complementary to rat $18 \mathrm{~S}$ ribosomal RNA (11) was hybridized to all filters in $0.9 \mathrm{M} \mathrm{NaCl}, 90 \mathrm{mM}$ sodium citrate, $1 \times$ Denhardt solution, $0.05 \%$ pyrophosphate, and $100 \mu \mathrm{g}$ yeast tRNA overnight at $37^{\circ} \mathrm{C}$. Filters were washed twice for $20 \mathrm{~min}$ at $45^{\circ} \mathrm{C}$ in $0.9 \mathrm{M} \mathrm{NaCl}, 90 \mathrm{mM}$ sodium citrate, and $0.05 \%$ pyrophosphate. To assure probe excess, a 15-fold molar excess of $18 \mathrm{~S}$ oligonucleotide was added (based on the calculated amount of $18 \mathrm{~S}$ ribosomal RNA loaded). Since ribosomal RNA is the predominant constituent of total cellular RNA, and the $18 \mathrm{~S}$ subunit represents a fixed proportion of ribosomal RNA, hybridization of this oligonucleotide was used as a measure of the total cellular RNA loaded for each sample. The size of the predominant band following $18 \mathrm{~S}$ hybridization corresponded to $18 \mathrm{~S}$ ribosomal RNA on ethidium bromide-stained RNA gels. Autoradiograms were scanned using an LKB 2222-010 Ultrascan XL laser densitometer with an LKB 2400 GelScan Software Package (LKB Instruments, Inc., Gaithersburg, MD). To estimate tissue ANF mRNA concentration, the ANF:18S ratio was determined by dividing the absorbance corresponding to ANF probe hybridization by the absorbance corresponding to $18 \mathrm{~S}$ probe hybridization.

Radioimmunoassay. Immunoreactive ANF (iANF) in guanidium isothiocyanate extracts of ventricular tissues (diluted 1:100) was measured by radioimmunoassay as previously described (12). The sensitivity of this assay was $95 \mathrm{pg}$ atriopeptin III equivalents per $\mathrm{ml}$ of diluted extract. Ventricular concentration of iANF was obtained by dividing the iANF by the tissue wet weight.

\section{Results}

Ventricular weights. The body weights of animals studied ranged from 361 to $454 \mathrm{~g}$ and ventricular weights were indexed accordingly. The control WKY animals studied demonstrated right and left ventricular to body weight ratios (Table I) similar to those previously characterized (13). BVH rats had severe biventricular hypertrophy, with both left and right ventricular to body weight ratios twice normal. This index of hypertrophy was statistically increased in BVH compared to WKY animals $(P<0.05)$, and was comparable to that documented in our initial report of BVH animals, in which a larger study population was examined (8).

Northern analysis. Northern blots using the rat ANF

Table I. Ventricular Weights and Hypertrophy Indices

\begin{tabular}{lllllll}
\hline Animal & Age & Wt & LV & LV/BW & RV & RV/BW \\
\hline & $m o$ & $g$ & $m g$ & & $m g$ & \\
WKY 1 & 13 & 454 & 952 & 2.10 & 226 & 0.50 \\
WKY 2 & 14 & 388 & 788 & 2.03 & 199 & 0.51 \\
WKY 3 & 14 & 438 & 842 & 1.92 & 256 & 0.58 \\
BVH 1 & 14 & 361 & 1076 & 2.98 & 449 & 1.2 \\
BVH 2 & 13 & 430 & 1885 & 4.39 & 463 & 1.1 \\
BVH 3 & 9 & 423 & 1538 & 3.36 & 485 & 1.2 \\
\hline
\end{tabular}

LV/BW (left ventricular weight to body weight) is an index of LV hypertrophy. A normal LV/BW ratio for WKY animals is $2.01 \pm 0.40$ (mean \pm standard deviation) (see reference 13).

$\mathrm{RV} / \mathrm{BW}$ (right ventricular weight to body weight) is an index of RV hypertrophy. A normal RV/BW ratio for WKY animals is $0.53 \pm 0.21$. $\mathrm{LV} / \mathrm{BW}$ and $\mathrm{RV} / \mathrm{BW}$ ratios in $\mathrm{BVH}$ animals are significantly greater than WKY ratios $(P<0.05)$ using a Student's $t$ test.

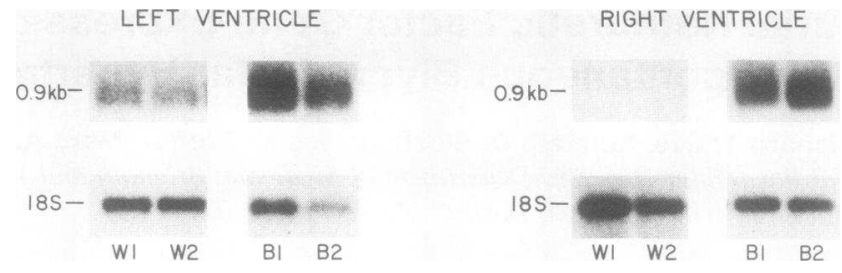

Figure 1. Northern analysis of rat ventricular RNA. RNA was prepared from ventricles of WKY (W1, W2) and BVH (B1, B2) rats and hybridized to a ${ }^{32} \mathrm{P}$-labeled Pst 1 restriction fragment derived from a rat ANF cDNA. Autoradiographs were exposed for $6 \mathrm{~h}$ on Kodak XAR film without an enhancing screen. The probe was then removed and the filters were hybridized to a ${ }^{32} \mathrm{P}$-labeled oligonucleotide complementary to RNA of the rat $18 \mathrm{~S}$ ribosomal subunit. Approximately $2.5 \mu \mathrm{g}$ of total cellular RNA was loaded on each lane.

cDNA probe identified a 0.9-kb mRNA species in all left ventricles (Fig. 1, Table II). The left ventricles of BVH rats had sixfold greater ANF mRNA concentrations as compared to the control animals. A low level of ANF mRNA was detected in the right ventricle of one control rat. Northern analysis of right ventricular ANF mRNA revealed a striking increase in the BVH rats. The ANF:18S ratios in BVH right ventricles approximated left ventricular ratios in hypertrophied animals. The ANF:18S ratios in right and left ventricles of BVH animals was statistically greater than that found in WKY controls. No apparent differences were discerned between left or right atrial ANF mRNA concentrations from individual animals (data not shown). Total atrial ANF mRNA levels were equivalent in WKY and BVH animals, and approximately 50 -fold greater than left ventricular concentrations of WKY animals.

Table II. Ventricular ANF MRNA:18S Ratios and Immunoreactive $A N F$

\begin{tabular}{|c|c|c|c|c|}
\hline & \multicolumn{2}{|c|}{ Left ventricle } & \multicolumn{2}{|c|}{ Right ventricle } \\
\hline & ANF:18S* & iANF $^{\ddagger}$ & ANF:18S* & iANF $^{\ddagger}$ \\
\hline WKY 1 & 0.3 & $240(228)$ & ND & $520(117)$ \\
\hline WKY 2 & 0.2 & $330(260)$ & ND & $290(58)$ \\
\hline WKY 3 & 0.3 & $273(230)$ & 0.1 & $119(31)$ \\
\hline BVH 1 & 1.4 & $1,092(1,175)$ & 1.9 & $860(386)$ \\
\hline BVH 2 & 2.1 & $551(1,039)$ & 1.2 & $1,020(473)$ \\
\hline BVH 3 & 2.2 & $540(830)$ & 1.6 & 781 (379) \\
\hline
\end{tabular}

* ANF:18S ratio was determined by dividing the absorbance of the ANF band by the absorbance of the $18 \mathrm{~S}$ band, and is expressed in arbitrary units. Left and right ventricular ANF:18S ratios were significantly greater in BVH animals compared to WKY animals $(P$ $<0.01$ ) based on Student's $t$ test. WKY 3 and BVH 3 were analyzed separately from those of WKY 1, 2 and BVH 1, 2.

‡ iANF concentration expressed as nanograms of atriopeptin III equivalents per gram of tissue, wet weight. Values in parentheses indicate the total content of iANF (in micrograms) per ventricle. Left ventricular iANF was not significantly different in BVH and WKY animals $(P>0.05)$, while right ventricular iANF significantly increased in BVH animals $(P<0.01)$ based on a Student's $t$ test. ND, not detected. 
Radioimmunoassay. Immunoreactive ANF (Table II) was detected in the left ventricles of both WKY and BVH rats. The concentration of iANF in BVH left ventricles was only threefold greater than in WKY controls, an increase which was not statistically significant $(P>0.05)$. The concentration of iANF detected in the right ventricles of animal WKY 1 was just above the sensitivity of the assay. All BVH animals exhibited right ventricular iANF concentrations comparable to those in the hypertrophied left ventricles.

\section{Discussion}

The BVH rat develops hypertrophy of both ventricles in response to an increased shunt fraction of the cardiac output; $\sim 45 \%$ of the cardiac output in these animals does not flow through capillary beds (8). To maintain oxygen delivery, these animals must increase cardiac output, leading to a naturally occurring hypertrophy of both ventricles. Our results demonstrate that these events also cause marked increases of ANF mRNA levels in the right and left ventricle.

While left ventricular ANF gene induction has been demonstrated by several laboratories $(6,7)$, high levels of right ventricular ANF gene expression have not previously been seen in experimental models of cardiac hypertrophy. Our data show that right ventricular induction of the ANF gene can occur, and at levels comparable to that seen in the left ventricle. The type and chronicity of hemodynamic changes produced in each model may be critical for chamber-specific expression of the ANF gene.

Spontaneous biventricular hypertrophy varies in several respects from previously reported experimental models of hypertrophy. First, this genetic condition causes gradual progression of the myopathy, with minimal acute deleterious effects. Animals were evaluated in this study at 9-14 mo of age. Other studies have assessed ANF gene induction shortly after an acute hemodynamic burden is placed on the animals. Precipitous changes in afterload, circulatory volume or arteriovenous shunting will primarily produce changes in left ventricular hemodynamic parameters. Involvement of the right ventricle is usually a gradual process, which would not be expected to be seen in the time course of acute studies.

If ANF expression by ventricular tissues occurs concordantly with hypertrophy, then experimental models producing primarily left ventricular hypertrophy would not be expected to induce right ventricular ANF gene expression. The BVH model of cardiac hypertrophy causes alterations of both ventricular chambers, and the ventricular weight to body weight index showed greater hypertrophy of the RV than LV (Table I). Concurrently, ANF mRNA was sixfold greater in the left ventricle of BVH animals as compared to WKY controls, but strikingly greater increases of ANF transcripts were found in hypertrophied right ventricles.

The immunoreactive ANF detected in ventricular chambers of WKY animals is low, and approximately 400 -fold less than that found in adult atria (12). Ventricular concentrations of ANF increased with hypertrophy, but less than anticipated given the increases in mRNA transcripts (Table II). We have previously demonstrated that ventricular cells in culture release ANF peptide constitutively and lack secretory granules characteristic of atrial cardiocytes, which store the peptide be- fore secretion (12). We conclude that hypertrophied ventricular tissues also secrete ANF predominantly by a constitutive pathway, and do not store the peptide before release.

High levels of ANF gene transcription in animals with well-compensated cardiac hypertrophy suggest two possible relationships between this peptide hormone and pathologic states. The normal heart responds to acute hemodynamic burdens with several compensatory mechanisms; increased preload to optimize myofilament overlap and function (FrankStarling mechanism), increased catecholamines to augment contractility, and myocardial hypertrophy to increase the mass of contractile tissue (14). Ventricular induction of a gene encoding an endogenous vasorelaxant, diuretic peptide might suggest that ANF participates in this adaptive response. Conversely, high level biventricular ANF gene expression in normotensive, euvolemic animals with spontaneous hypertrophy might imply that this gene is only a marker of the multifaceted cellular changes accompanying hypertrophy, rather than an adaptive response to acute hemodynamic stress.

The study of ventricular hypertrophy has relied largely on the experimental production of pressure or volume overload. BVH rats provide a naturally occurring model of spontaneous hypertrophy without acute hemodynamic perturbations. Definition of gene activation in this model may lead to a clearer understanding of those factors that are associated with the pathologic state of cardiac hypertrophy from those compensatory responses that are critical for restoration of hemodynamic homeostasis. Further studies on the role of ANF in this model should increase our knowledge of this hormone's function in physiologic and pathologic states.

\section{Acknowledgments}

We thank J. G. Seidman for helpful discussions and Gary Gryan for synthesis of the oligonucleotide.

This work was supported by National Institutes of Health grants HL-36074, HL-35642, and HL-19259. Dr. Lee is a recipient of a Physician Scientist Award (HL-01835) from the National Heart, Lung, and Blood Institute. Dr. Bloch is a recipient of a Pfizer Pharmaceuticals Postdoctoral Fellowship. Dr. J. Pfeffer is a recipient of a Research Career Development Award (HL-01723) from the National Heart, Lung, and Blood Institute. Dr. Seidman is a recipient of an American Heart Association Clinician-Scientist Award.

\section{References}

1. de Bold, A. J. 1985. Atrial natriuretic factor: a hormone produced by the heart. Science (Wash. DC). 230:767-770.

2. Needleman, P. 1986. The expanding physiological roles of atrial natriuretic factor. Nature (Lond.) 321:199-200.

3. Lang, R. E., H. Tholken, D. Ganten, R. C. Luft, H. Rushoaho, and T. Unter. 1985. Atrial natriuretic factor-a circulatory hormone stimulated by volume loading. Nature (Lond.) 314:264-266.

4. Gardner, D. G., C. F. Deschepper, W. F. Ganong, S. Hane, J. Fiddes, J. D. Baxter, and J. Lewicki. 1986. Extra-atrial expression of the gene for atrial natriuretic factor. Proc. Natl. Acad. Sci. USA. 83:6697-6701.

5. Nemer, M., J. P. Lavigne, J. Drouin, G. Thibault, M. Gannon, and T. Antakly. 1986. Expression of atrial natriuretic factor in heart ventricular tissue. Peptides. 7:1147-1152.

6. Day, M. L., D. Schwartz, R. C. Wiegand, T. Stockmazn, S. R. Brunnert, H. E., Tolunay, M. G. Currie, D. G. Standaert, and P. 
Needleman. 1987. Ventricular atriopeptin: unmasking of messenger RNA and peptide synthesis by hypertrophy or dexamethasone.

Hypertension. 9:485-491.

7. Lattion, A. L., J. B. Michel, E. Arnauld, P. Corvol, and R. Soubrier. 1986. Myocardial recruitment during ANF mRNA increase with volume overload in the rat. Am. J. Physiol. 251:H890-H896.

8. Pfeffer, M. A., J. M. Pfeffer, F. G. Dunn, K. Nishiyama, M. Tsuchiya, and E. D. Frohlich. 1979. Natural biventricular hypertrophy in normotensive rats. I. Physical and hemodynamic characteristics. Am. J. Physiol. 5:H640-H643.

9. Chirgwin, J. M., A. E. Przybyla, R. F. MacDonald, and W. J. Rutter. 1979. Isolation of biologically active ribonucleic acid from sources enriched in ribonuclease. J. Biochem. 18:5294-5299.

10. Seidman, C. E., A. D. Duby, E. Choi, R. M. Graham, E. Haber, C. J. Homcy, J. A. Smith, and J. G. Seidman. 1984. The structure of the rat pre-proatrial natriuretic factor as defined by a complementary DNA clone. Science (Wash. DC) 225:324-326.

11. Chan, Y. L., R. Butell, J. F. Noller, and I. G. Wool. 1984. The nucleotide sequence of a rat $18 \mathrm{~S}$ ribosomal ribonucleic acid gene and a proposal for the secondary structure of $18 \mathrm{~S}$ ribosomal ribonucleic acid. J. Biol. Chem. 259:224-230.

12. Bloch, K. D., J. G. Seidman, J. D. Naftilan, J. T. Fallon, and C. E. Seidman. 1986. Neonatal atria and ventricles secrete atrial natriuretic factor via tissue-specific secretory pathways. Cell. 47:695-702.

13. Pfeffer, J. M., M. A. Pfeffer, M. C. Fishbein, and E. D. Frohlich. 1979. Cardiac function and morphology with aging in the spontaneously hypertensive rat. Am. J. Physiol. 237:H461-H468.

14. Braunwald, E. 1984. Pathophysiology of Heart Failure. In Heart Disease, A Textbook of Cardiovascular Medicine. E. Braunwald, editor. W. B. Saunders Co., Philadelphia. 447-466. 